\title{
State-Independent Uncertainty Relations and Entanglement Detection in Noisy Systems
}

\author{
René Schwonnek, ${ }^{*}$ Lars Dammeier, ${ }^{\dagger}$ and Reinhard F. Werner \\ Leibniz Universität Hannover-Institut für Theoretische Physik, Hannover 30167, Germany
}

(Received 31 May 2017; published 27 October 2017)

\begin{abstract}
Quantifying quantum mechanical uncertainty is vital for the increasing number of experiments that reach the uncertainty limited regime. We present a method for computing tight variance uncertainty relations, i.e., the optimal state-independent lower bound for the sum of the variances for any set of two or more measurements. The bounds come with a guaranteed error estimate, so results of preassigned accuracy can be obtained straightforwardly. Our method also works for postive-operator-valued measurements. Therefore, it can be used for detecting entanglement in noisy environments, even in cases where conventional spin squeezing criteria fail because of detector noise.
\end{abstract}

DOI: 10.1103/PhysRevLett.119.170404

Introduction.-Uncertainty relations quantitatively express a phenomenon which is ubiquitous in quantum mechanics: Given two observables $A$ and $B$, it is usually impossible to prepare a state such that the respective outcome distributions of these observables are both sharp. Of course, for the best known example of this, the position and momentum observables, the relation is in every textbook. It was first established by Kennard [1], who turned Heisenberg's heuristic ideas [2] into a quantitative statement. In particular, it was his idea to consider the variances [3] of momentum and position in the state $\rho$ as the mathematical expression of sharpness. Kennard's relation $\Delta_{\rho}^{2}(P) \Delta_{\rho}^{2}(Q) \geq \hbar^{2} / 4$ is tight; i.e., the constant on the righthand side is the best possible because it is attained for Gaussian pure states.

The aim of our Letter is to provide an efficient method to obtain the best possible bounds for any given pair of measurements $A, B$. This is of direct use in the increasing number of experiments that reach the uncertainty-limited regime. A particular application is the certification of entanglement via steering inequalities [4-6]. In such applications, even if one does not necessarily need an optimal bound, it is crucial to have a correct one, i.e., a bound valid for all states. Any algorithm based on computing the uncertainties "for sufficiently many states" will fail to guarantee this correctness. In particular, in highdimensional Hilbert spaces, typical states will not have uncertainties near the boundary, so it is actually hard to explore the set of uncertainty pairs $\left(\Delta_{\rho}^{2}(A), \Delta_{\rho}^{2}(B)\right)$ "from within." Our method uses instead an "outer" approximation, which has the virtue that in every step it provides a correct bound. The bound is iteratively improved, converging to the optimal one. This feature sets our method apart from several recent works, in which ad hoc methods were used to provide uncertainty bounds. The problem of getting optimal uncertainty bounds becomes more difficult as the dimension $d$ of the Hilbert space increases. Indeed, naively it would seem to be a search problem on the $2 d-2$ dimensional manifold of pure states, which in bad cases might scale exponentially with $d$. However, we can do much better. We reformulate the problem as a geometric problem in three dimensions, namely, of getting a sequence of outer polyhedral approximation of a certain convex set; see Fig. 1. Any such approximation gives a valid uncertainty bound. In the iteration step, i.e., for computing a tighter approximation, one has to compute the lowest eigenvalue of a certain Hermitian combination of the operators $A$ and $B$. Those eigenvalue problems now determine the scaling of our method as a function of dimension, which will be a low order polynomial in $d$. Moreover, if additional information is available about $A$ and $B$, for example, if they are both sparse in the same basis, eigenvalue computations can be speeded up considerably, and our method will speed up by the same factor.

Tight uncertainty bounds have only been obtained for a few specific pairs of observables. One example is angular momentum measurement, where bounds for two or three

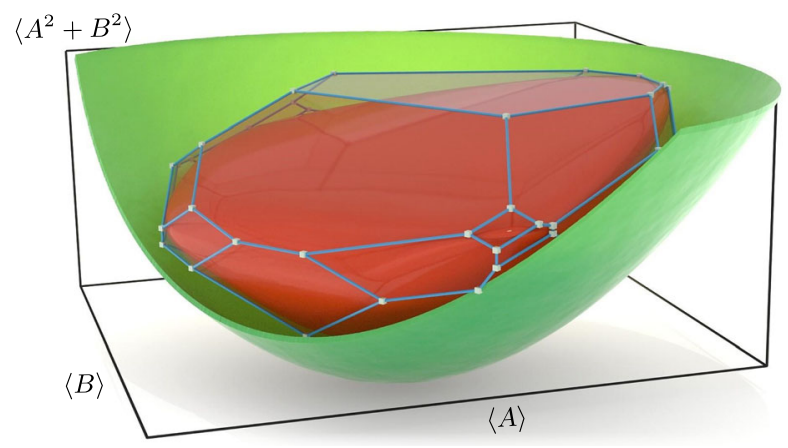

FIG. 1. Minimizing the sum of the variances of two observables $A$ and $B$ can be expressed entirely in terms of the set $\mathcal{C}$ of possible triples $\left(\langle A\rangle_{\rho},\langle B\rangle_{\rho},\left\langle A^{2}+B^{2}\right\rangle_{\rho}\right)$ (red solid convex body), namely, as finding that vertical displacement of the surface $z=x^{2}+y^{2}$ (green paraboloid) which just touches $\mathcal{C}$ from below. We successively approximate $\mathcal{C}$ by polytopes (blue edges, boxed vertices) from the outside, and perform the minimization on this polytope. This gives a converging sequence of correct stateindependent uncertainty relations. 
orthogonal spin components $[4,7,8]$ are known. In those cases symmetry crucially helps to reduce the problem. Other examples are qubits [9], for which the low dimension allows an analytical solution.

There are also variants, in which the sharpness of a distribution is measured by other quantities than the usual variance [10-13], for instance, entropies [14-25] and its generalization to majorization uncertainty relations [26-28], or where more than two observables are considered simultaneously [29-31]. However, in none of those cases a general method for obtaining optimal bounds in known.

Methods.-Linear state independent bounds: Since we are interested in state-independent bounds [32] we have no use for the often-cited general relation by Robertson [33] (and its improvements [34]), which have a state-dependent expression like $\langle i[A, B]\rangle_{\rho}^{2}$, or similar, on the right-hand side. Indeed, any relation of product form $\Delta_{\rho}^{2}(A) \Delta_{\rho}^{2}(B) \geq c$ is useless for state-independent relations in the finite dimension: $A$ and $B$ have discrete eigenvalues, so the trivial $c=0$ is the best possible bound. We therefore consider bounds of the form

$$
\Delta_{\rho}^{2}(A)+\Delta_{\rho}^{2}(B) \geq c .
$$

Here, $c$ is the largest constant for which the above holds on any quantum state $\rho$. Since our method handles arbitrary $A$ and $B$ we can also admit factors here, i.e., inequalities of the form $\lambda \Delta_{\rho}^{2}(A)+\mu \Delta_{\rho}^{2}(B) \geq c(\lambda, \mu)$. Each of these constrains the set of uncertainty pairs $\left(\Delta_{\rho}^{2}(A), \Delta_{\rho}^{2}(B)\right)$ to a half-plane, and together they outline the uncertainty set (or, more precisely its "lower convex hull," see Fig. 4 and Refs. [7,9,35]).

To see the connection to eigenvalue problems, we write the optimal constant in Eq. (1) as

$$
c=\min _{\rho} \min _{a, b}\left\langle(A-a \mathbb{1})^{2}+(B-b \mathbb{1})^{2}\right\rangle_{\rho} .
$$

Here we just wrote the variance as the minimal quadratic deviation, using that the minimum with respect to $a$ is attained at the expectation $a=\langle A\rangle_{\rho}$. On the other hand, if we fix $a$ and $b$, the minimization with respect to $\rho$ is exactly the ground state problem for the operator in parentheses. This suggested our previous ansatz [7], which we call the seesaw algorithm: One alternatingly minimizes with respect to $\rho$ and $(a, b)$. In many practical cases this converges quickly, and with the safeguard of trying out several initial values it seems fairly reliable. However, in general the method of alternating minimization may easily fail to find the global minimum, and there is no proof of convergence. Intermediate results of the seesaw algorithm give an upper bound on $c$, but as an upper bound on a lower bound this is useless for applications. Moreover, there are indications that the seesaw algorithm actually may get trapped.

Geometry of outer approximations.-In contrast, the method described in this Letter is an outer method, in which all intermediate steps give valid lower and upper bounds on $c$. Its geometric core is the joint numerical range

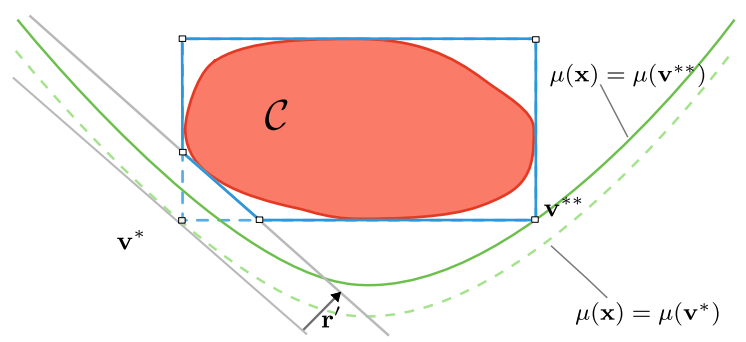

FIG. 2. Two dimensional sketch of geometry and the basic algorithm: The set $\mathcal{C}$ (red) with its outer approximation $\mathcal{P}(\mathcal{R})$ (blue and blue dashed lines) and the extremal points $\mathcal{E}(\mathcal{R})$ (white squares). By adding the direction $\mathbf{r}^{\prime}$, the polyhedral approximation is refined and the lower bound $c_{-}(\mathcal{R})$ is improved from $\mu\left(\mathbf{v}^{*}\right)$ (dashed green parabola) to $\mu\left(\mathbf{v}^{* *}\right)$ (green parabola).

$$
\mathcal{C}=\left\{\left(\langle A\rangle_{\rho},\langle B\rangle_{\rho},\left\langle A^{2}+B^{2}\right\rangle_{\rho}\right) \mid \rho \in \mathcal{S}(\mathcal{H})\right\}
$$

where $\mathcal{S}(\mathcal{H})$ denotes the state space, i.e., the set of density operators. Notice first that this set contains all the information necessary to compute $c$ from Eq. (2). With the quadratic functional $\mu(\mathbf{x}):=z-x^{2}-y^{2}$ of $\mathbf{x}=(x, y, z) \in$ $\mathbb{R}^{3}$ we find

$$
c=\min _{\rho \in \mathcal{S}(\mathcal{H})} \Delta_{\rho}^{2}(A)+\Delta_{\rho}^{2}(B)=\min _{\mathbf{x} \in \mathcal{C}} \mu(\mathbf{x}) .
$$

Now the set $\mathcal{C}$ is clearly convex and compact, because the state space $\mathcal{S}(\mathcal{H})$ has these properties, and they are preserved by the map taking $\rho$ to the tuple of expectations. The set $\mathcal{C}$ is, therefore, completely described by the linear inequalities it satisfies. To get such inequalities, let $\mathbf{r}=$ $\left(r_{1}, r_{2}, r_{3}\right)$ be a real vector, and consider $H(\mathbf{r})=r_{1} A+$ $r_{2} B+r_{3}\left(A^{2}+B^{2}\right)$. Let $h(\mathbf{r})$ denote the smallest eigenvalue of this operator. Then, for any state $\rho$, and, hence, the corresponding tuple $\mathbf{x} \in \mathcal{C}$ of expectations:

$$
\mathbf{r} \cdot \mathbf{x}=\langle H(\mathbf{r})\rangle_{\rho} \geq h(\mathbf{r}) .
$$

Now let $\mathcal{R} \subset \mathbb{R}^{3}$ be any finite set of vectors, and consider the polytope $\mathcal{P}(\mathcal{R})$ of those points $\mathbf{x}$, which just satisfy the inequalities (5) with $\mathbf{r} \in \mathcal{R}$. Since these vectors satisfy fewer constraints than $\mathcal{C}$, we have $\mathcal{C} \subset \mathcal{P}(\mathcal{R})$; i.e., this is an outer approximation of $\mathcal{C}$. Denote by $\mathcal{E}(\mathcal{R})$ the set of extreme points of $\mathcal{P}(\mathcal{R})$, which is also finite. Then

$$
c \geq \min _{\mathbf{x} \in \mathcal{P}(\mathcal{R})} \mu(\mathbf{x})=\min _{\mathbf{x} \in \mathcal{E}(\mathcal{R})} \mu(\mathbf{x})=: c_{-}(\mathcal{R}) .
$$

Here we have used, first, that the minimum over a larger set is smaller, and, second, that the functional $\mu$ is concave, so that the minimum over a compact convex set is attained at an extreme point. Hence, for every finite set $\mathcal{R}$ of directions, we get a lower bound on $c$, which is computed as a finite minimum over $\mathcal{E}(\mathcal{R})$. On the other hand, for each $r \in \mathcal{R}$ we get a point $\mathbf{x}^{*}(r)$, with equality in Eq. (5). Then 


$$
c \leq \min _{\mathbf{r} \in \mathcal{R}} \mu\left(\mathbf{x}^{*}(\mathbf{r})\right)=: c_{+}(\mathcal{R}) .
$$

So for every set $\mathcal{R}$, this procedure estimates the optimal constant $c$ up to a precision $\varepsilon=c_{+}(\mathcal{R})-c_{-}(\mathcal{R})$.

Basic algorithm.-The idea of the algorithm is now to let the set $\mathcal{R}$ grow step by step, which shrinks $\mathcal{P}(\mathcal{R})$, so $c_{-}(\mathcal{R})$ increases and $c_{+}(\mathcal{R})$ decreases (see Figs. 2 and 3 ). The algorithm terminates when $\varepsilon$ is below the target accuracy.

Apart from the set $\mathcal{R}$ it is useful to keep track of the polytope $\mathcal{P}(\mathcal{R})$ in the form of a list of vertices $\mathcal{E}(\mathcal{R})$ and edges. To arrive at the next approximation $\mathcal{R}^{\prime}=\mathcal{R} \cup\left\{\mathbf{r}^{\prime}\right\}$ : (1) Determine a vertex $\mathbf{v}^{*} \in \mathcal{E}(\mathcal{R})$ at which $\mu$ becomes minimal, and set

$$
\mathbf{r}^{\prime}=\left.\nabla \mu\right|_{\mathbf{v}^{*}}
$$

(2) Solve the minimum-eigenvalue problem for $H\left(\mathbf{r}^{\prime}\right)$. This provides the bound $h\left(\mathbf{r}^{\prime}\right)$ for the new inequality (5), and an expectation tuple $\mathbf{x}^{*}$ corresponding to the ground state. (3) Compute $\mu\left(\mathbf{x}^{*}\right)$ and update $c_{+}\left(\mathcal{R}^{\prime}\right)$, if this is smaller than the current value. (4) Take the new inequality (5), and compute the intersections with all current edges of $\mathcal{P}(\mathcal{R})$. This will give some new extreme points for $\mathcal{E}\left(\mathcal{R}^{\prime}\right)$, and corresponding edges. (5) Evaluate $\mu$ on the new extreme points in $\mathcal{E}\left(\mathcal{R}^{\prime}\right)$ and update $c_{-}\left(\mathcal{R}^{\prime}\right)$. Terminate if $c_{+}\left(\mathcal{R}^{\prime}\right)-$ $c_{-}\left(\mathcal{R}^{\prime}\right)$ is as small as desired. Otherwise go to step 1.
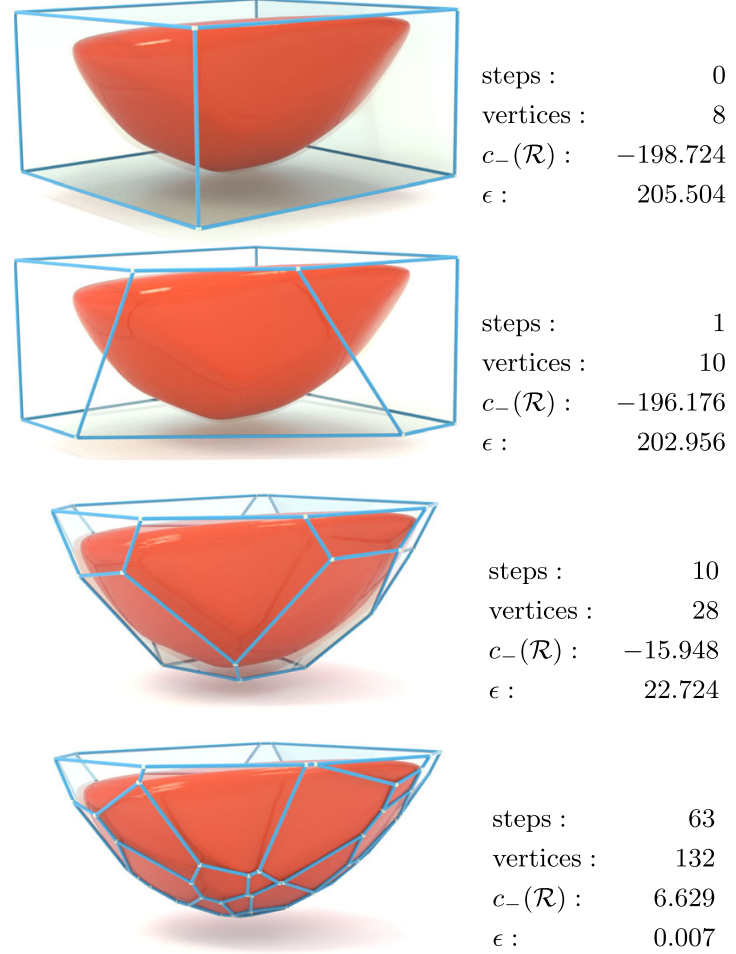

$$
\begin{array}{lr}
\text { steps : } & 63 \\
\text { vertices : } & 132 \\
c_{-}(\mathcal{R}): & 6.629 \\
\epsilon: & 0.007
\end{array}
$$

FIG. 3. Improving the outer approximation of $\mathcal{C}$ (red convex body) by adding more directions to the set $\mathcal{R}$. Every direction $\mathbf{r} \in \mathcal{R}$ gives a face of $\mathcal{P}(\mathcal{R})$ (blue polytope). New directions are chosen such that the vertex with the lowest value of $\mu$ will be cut off. Example generated from randomly chosen $A, B \in \mathbb{R}^{10 \times 10}$.
All these steps except the choice in step 1 are dictated by the geometry of outer approximation. The rationale of the choice (8) (apart from its flavor of gradient search) is that, whenever possible, it will eliminate the vertex $\mathbf{v}^{*}$ from $\mathcal{P}\left(\mathcal{R}^{\prime}\right)$, and thus strictly increase $c_{-}(\mathcal{R})$, unless there are other vertices with the same value of $\mu$, which have first to be eliminated in a similar manner. A proof of this statement is provided in the Supplemental Material [36]. As an application of our method, we derived the uncertainty relations for two nonorthogonal spin components; see the Supplemental Material [36].

Generalization to POVMs.-Our method can be applied with minimal modifications to generalized measurements, i.e., observables given by positive operator valued measures (POVMs). In general, a POVM measurement $\mathcal{A}$ is described by its outcomes $\left\{a_{i}\right\}$ and corresponding effects $\left\{E_{i}\right\}[37,38]$, where the probability of obtaining the outcome $a_{i} \in \mathbb{R}$ is given by $\operatorname{tr}\left(\rho E_{i}\right)$. The moments of an outcome distribution are then given by the expectations of the moment operators $A^{(n)}=\sum_{i}\left(a_{i}\right)^{n} E_{i}$. The only difference from the "standard" projection valued case is that the identity $A^{(n)}=\left(A^{(1)}\right)^{n}$ no longer holds. But this is not required for our method.

We therefore only need to express variances as $\Delta_{\rho}^{2}(A)=\left\langle A^{(2)}\right\rangle_{\rho}-\left\langle A^{(1)}\right\rangle_{\rho}^{2}$, and replace in Eq. (3) and the definition of $H(\mathbf{r}): A^{2}$ by $A^{(2)}, A$ by $A^{(1)}$, and analogously for $B$.

Application to entanglement detection.-In Refs. [4,5], it was shown that every state-independent uncertainty relation like Eq. (4) yields a nonlinear entanglement witness, when applied to local measurements in a bipartition. Here the following scenario is considered: Two parties, Alice and Bob, can perform local measurements $A_{1}, A_{2}$ such as $B_{1}$, $B_{2}$, on an unknown quantum state $\rho$. Their goal is to decide if $\rho$ is entangled or not. For this, they measure the "sum observables" $M_{1}, M_{2}$, given by

$$
M_{i}=A_{i} \otimes \mathbb{1}+\mathbb{1} \otimes B_{i} .
$$

In the POVM case this is generalized to measuring $A_{i}$ on Alice's side, $B_{i}$ on Bob's, and adding the outcomes, which results in

$$
\begin{gathered}
M_{i}^{(1)}=A_{i}^{(1)} \otimes \mathbb{1}+\mathbb{1} \otimes B_{i}^{(1)}, \\
M_{i}^{(2)}=A_{i}^{(2)} \otimes \mathbb{1}+2 A_{i}^{(1)} \otimes B_{i}^{(1)}+\mathbb{1} \otimes B_{i}^{(2)} .
\end{gathered}
$$

Now if $\rho=\rho_{A} \otimes \rho_{B}$ is uncorrelated, variances just add up, so

$$
\Delta_{\rho}^{2}\left(M_{1}\right)+\Delta_{\rho}^{2}\left(M_{2}\right) \geq c_{A}+c_{B},
$$

where $c_{A}$ and $c_{B}$ are the optimal uncertainty constants for the observable pairs $\left(A_{1}, A_{2}\right)$ and $\left(B_{1}, B_{2}\right)$, respectively. Since the variance is concave, this inequality holds also for 


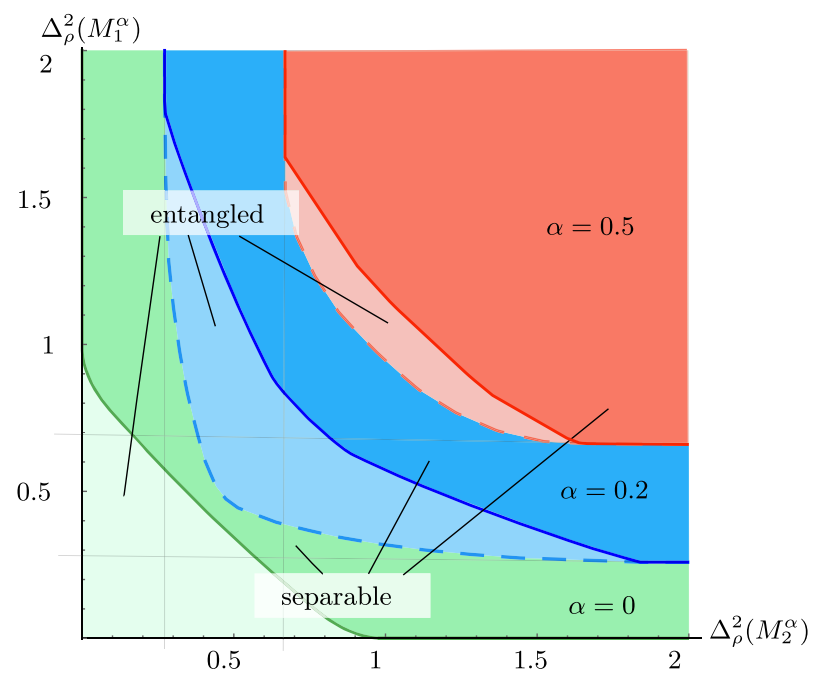

FIG. 4. Uncertainty regions for entangled and separable states. Superposition of the graphs for different noise levels $\alpha$ : green $=0$, blue $=0.2$, red $=0.5$. In this example we consider local measurements of orthogonal spin-1 components, i.e., $M_{i}=L_{i}^{A}+L_{i}^{B}$.

all convex combinations of uncorrelated states, i.e., for all separable states [4].

Hence, if Eq. (12) is violated, $\rho$ must be entangled. Of course, there is also an uncertainty bound $c_{M}$ for the observable pair $\left(M_{1}, M_{2}\right)$. So the interesting range allowing the conclusion " $\rho$ is entangled" is marked by

$$
c_{A}+c_{B}>\Delta_{\rho}^{2}\left(M_{1}\right)+\Delta_{\rho}^{2}\left(M_{2}\right) \geq c_{M} .
$$

For angular momentum measurements, Eq. (12) is a spin-squeezing [39] criterion. As such, it requires the same experimental data as other spin-squeezing criteria, see Refs. [40,41], namely, only a measurement of first and second moments of the total angular momentum. In contrast to entanglement criteria based on tomography, these are advantageous in typical experimental implementation. A popular example are collective spins of many particle systems [42], where measurements on BECs were performed.

We further sharpen this criterion by applying it to the observable pairs $\left(\mu A_{1}, \lambda A_{2}\right)$ and $\left(\mu B_{1}, \lambda B_{2}\right)$. In this way we get two convex regions of pairs $\left(\Delta_{\rho}^{2}\left(M_{1}\right), \Delta_{\rho}^{2}\left(M_{2}\right)\right)$ : A larger one containing the pairs achievable with arbitrary states, given by the bounds of the type $c_{M}$, and a smaller one attainable by separable states, given by the bounds of the type $c_{A}+c_{B}$. As Fig. 4 shows, this increases the parameter range for which entanglement can be certified. The linear uncertainty bound with equal weights as a function of the local noise, evaluated for measurements $M_{1}$ and $M_{2}$ on separable and entangled states is shown in the Supplemental Material [36].

Entanglement detection with noisy detectors. - The generalization to POVMs increases the possibilities for entanglement detection. Suppose for the sake of discussion that before hitting the detector each subsystem goes through a known noisy channel. This typically increases variance [43], so traditional spin squeezing inequalities would often fail to detect entanglement. Indeed the state after the action of the noisy channels may well fail to be entangled. On the other hand, we might be interested in the presence of entanglement before the action of the noise. This is the appropriate view when the noise is inherent in the detection process. The noise is thus applied in the Heisenberg picture, turning even a standard projection valued measurement into a proper POVM. This might easily find entanglement, which would go undetected by a direct application of the spin squeezing criterion.

These possibilities are shown in Fig. 4 by superimposing the entanglement detection regions for three different noise levels of a partially depolarizing channel $\rho \mapsto$ $(1-\alpha) \rho+\alpha \rho_{0}$, where $\rho_{0} \propto \mathbb{1}$ is the maximally mixed state, and $\alpha$ is a noise parameter. Increasing $\alpha$ shifts the diagram towards larger variances, but even for a modest noise level of $\alpha=0.2$ the entanglement detection region lies entirely in the region where traditional spin squeezing (corresponding to $\alpha=0$ ) would never find any entanglement.

Conclusions and outlook.-We provided an algorithm for determining the optimal uncertainty bounds for two arbitrary observables. The precision of the bound is controlled as a duality gap, so terminating the iteration at any step gives a certified lower uncertainty bound together with an error estimate.

The method can, in principle, be extended to more observables, or to variances based not on quadratic but higher order deviations. However, this would increase the dimension of the geometric problem. Thus, at every new approximation step one has to determine the intersection of the polytope with the new supporting hyperplane. This requires a better bookkeeping of the topological structure of the polytopes, and a local version of the vertex enumeration problem [44].

The inequalities derived here have an immediate application to entanglement detection by generalized spin squeezing criteria. The possibility to use arbitrary observables (rather than orthogonal angular momentum components) greatly increases the versatility of this method.

It is an apparently open problem how strong the method becomes with arbitrary $A_{i}, B_{j}$, i.e., is every entangled state violating a local uncertainty relation. The problem has been studied carefully for orthogonal spin components [5,6], but we do not know of a characterization of the (un-)detectable, possibly entangled states.

We gratefully acknowledge inspiring conversations and email exchange with Marcus Cramer, Otfried Gühne, Géza Tóth, Kais Abdelkhalek, David Reeb and Terry Farrelly. We also acknowledge financial support from the RTG 1991 and CRC 1227 DQ-mat funded by the DFG and the collaborative research project Q.com-Q funded by the BMBF. 
*rene.schwonnek@itp.uni-hannover.de

${ }^{\dagger}$ lars.dammeier@itp.uni-hannover.de

*reinhard.werner@itp.uni-hannover.de

[1] E. Kennard, Zur Quantenmechanik einfacher Bewegungstypen, Z. Phys. 44, 326 (1927).

[2] W. Heisenberg, Über den anschaulichen Inhalt der quantentheoretischen Kinematik und Mechanik, Z. Phys. 43, 172 (1927).

[3] For Hermitian operators we define the variance of a state $\rho$ by $\Delta_{\rho}^{2}(A)=\left\langle A^{2}\right\rangle_{\rho}-\langle A\rangle_{\rho}^{2}=\operatorname{tr} \rho A^{2}-(\operatorname{tr} \rho A)^{2}$.

[4] H.F. Hofmann and S. Takeuchi, Violation of local uncertainty relations as a signature of entanglement, Phys. Rev. A 68, 032103 (2003).

[5] O. Gühne and G. Tóth, Entanglement detection, Phys. Rep. 474, 1 (2009).

[6] O. Gühne, Ph.D. thesis, Universität Hannover, 2004.

[7] L. Dammeier, R. Schwonnek, and R. F. Werner, Uncertainty relations for angular momentum, New J. Phys. 17, 093046 (2015).

[8] Q. He, S.-G. Peng, P. D. Drummond, and M. D. Reid, Planar quantum squeezing and atom interferometry, Phys. Rev. A 84, 022107 (2011).

[9] A. A. Abbott, P.-L. Alzieu, M. J. W. Hall, and C. Branciard, Tight state-independent uncertainty relations for qubits, Math. Mag. 4, 8 (2016).

[10] P. Busch, P. Lahti, and R. F. Werner, Measurement uncertainty relations, J. Math. Phys. (N.Y.) 55, 042111 (2014).

[11] R. F. Werner, Uncertainty relations for general phase spaces, Front. Phys. 11, 110305 (2016).

[12] P. Busch, J. Kiukas, and R. F. Werner, Sharp uncertainty relations for number and angle, arXiv:1604.00566.

[13] Y. Huang, Variance-based uncertainty relations, Phys. Rev. A 86, 024101 (2012).

[14] H. Maassen and J. Uffink, Generalized Entropic Uncertainty Relations, Phys. Rev. Lett. 60, 1103 (1988).

[15] K. Abdelkhalek, R. Schwonnek, H. Maassen, F. Furrer, J. Duhme, P. Raynal, B.-G. Englert, and R. F. Werner, Optimality of entropic uncertainty relations, Int. J. Quantum. Inform. 13, 1550045 (2015).

[16] P. Coles, L. Yu, and M. Zwolak, Relative entropy derivation of the uncertainty principle with quantum side information, arXiv:1105.4865.

[17] J. Sánchez-Ruiz, Improved bounds in the entropic uncertainty and certainty relations for complementary observables, Phys. Lett. A 201, 125 (1995).

[18] J. Sánches-Ruiz, Optimal entropic uncertainty relation in twodimensional Hilbert space, Phys. Lett. A 244, 189 (1998).

[19] G. C. Ghirardi, L. Marinatto, and R. Romano, An optimal entropic uncertainty relation in a two-dimensional Hilbert space, Phys. Lett. A 317, 32 (2003).

[20] R. Adamczak, R. Latała, Z. Puchała, and K. Życzkowski, Asymptotic entropic uncertainty relations, J. Math. Phys. (N.Y.) 57, 032204 (2016).

[21] P. Coles and F. Furrer, State-dependent approach to entropic measurement-disturbance relations, Phys. Lett. A 379, 105 (2015).

[22] P. Coles, R. Colbeck, L. Yu, and M. Zwolak, Uncertainty Relations from Simple Entropic Properties, Phys. Rev. Lett. 108, 210405 (2012).

[23] B.-G. Englert, D. Kaszlikowski, L. C. Kwek, and W. H. Chee, Wave-particle duality in multi-path interferometers:
General concepts and three-path interferometers, Int. J. Quantum. Inform. 06, 129 (2008).

[24] P. Coles, M. Berta, M. Tomamichel, and S. Wehner, Entropic uncertainty relations and their applications, Rev. Mod. Phys. 89, 015002 (2017).

[25] A. E. Rastegin, Rényi formulation of the entropic uncertainty principle for POVMs, J. Phys. A 43, 155302 (2010).

[26] Ł. Rudnicki, Z. Puchała, and K. Życzkowski, Strong majorization entropic uncertainty relations, Phys. Rev. A 89, 052115 (2014).

[27] Z. Puchała, Ł. Rudnicki, and K. Życzkowski, Majorization entropic uncertainty relations, J. Phys. A 46, 272002 (2013).

[28] S. Friedland, V. Gheorghiu, and G. Gour, Universal Uncertainty Relations, Phys. Rev. Lett. 111, 230401 (2013).

[29] S. Kechrimparis and S. Weigert, Heisenberg uncertainty relation for three canonical observables, Phys. Rev. A 90, 062118 (2014).

[30] S. Kechrimparis and S. Weigert, Preparational uncertainty relations for $n$ continuous variables, Math. Mag. 4, 49 (2016).

[31] J. Kaniewski, M. Tomamichel, and S. Wehner, Entropic uncertainty from effective anticommutators, Phys. Rev. A 90, 012332 (2014).

[32] D. Deutsch, Uncertainty in quantum measurements, Phys. Rev. Lett. 50, 631 (1983).

[33] H. P. Robertson, The uncertainty principle, Phys. Rev. 34, 163 (1929).

[34] L. Maccone and A. K. Pati, Stronger Uncertainty Relations for all Incompatible Observables, Phys. Rev. Lett. 113, 260401 (2014).

[35] R. Schwonnek, D. Reeb, and R. F. Werner, Measurement uncertainty for finite quantum observables, Math. Mag. 4, 38 (2016).

[36] See Supplemental Material at http://link.aps.org/ supplemental/10.1103/PhysRevLett.119.170404 for strict monoticity of the gap, speed of the numerical convergence, applications to non orthogonal spin-components, and entanglement detection in dependence of local noise.

[37] G. Ludwig, Foundations of Quantum Mechanics I (Springer, Berlin, Heidelberg, 1983).

[38] T. Heinosaari and M. Ziman, The Mathematical Language of Quantum Theory: From Uncertainty to Entanglement (Cambridge University Press, Cambridge, England, 2011).

[39] J. Ma, X. Wang, C.-P. Sun, and F. Nori, Quantum spin squeezing, Phys. Rep. 509, 89 (2011).

[40] A. S. Sørensen, L. Duan, I. Cirac, and P. Zoller, Manyparticle entanglement with Bose-Einstein condensates, Nature (London) 409, 63 (2001).

[41] A. S. Sørensen and K. Mølmer, Entanglement and extreme spin squeezing, Phys. Rev. Lett. 86, 4431 (2001).

[42] B. Lücke, J. Peise, G. Vitagliano, J. Arlt, L. Santos, G. Tóth, and C. Klempt, Detecting multiparticle entanglement of dicke states, Phys. Rev. Lett. 112, 155304 (2014).

[43] B. Lücke, M. Scherer, J. Kruse, L. Pezzé, F. Deuretzbacher, P. Hyllus, O. Topic, J. Peise, W. Ertmer, J. Arlt, L. Santos, A. Smerzi, and C. Klempt, Twin matter waves for interferometry beyond the classical limit, Science 334, 773 (2011).

[44] D. Avis and K. Fukuda, A pivoting algorithm for convex hulls and vertex enumeration of arrangements and polyhedra, Discrete Comput. Geom. 8, 295 (1992). 